\title{
CONTRIBUCIÓN AL DEBATE SOBRE LA VOCACIÓN ESPACIAL RURAL. UN ENFOQUE SOBRE EL MODELO RESIDENCIAL EJIDAL DEL VALLE DE MEXICALI ${ }^{1}$
}

\author{
Por \\ Djamel Toudert*
}

\begin{abstract}
RESUMEN
¿Tenemos los medios para hacer dos políticas, una urbana y otra rural? ¿Podemos considerar la salvación del mundo rural dentro de una alternativa diferente a la de la política agricola? En este trabajo, más que aportar soluciones, se plantean diversos cuestionamientos; el propósito es reflexionar sobre el debate de las mutaciones espaciales que afectan al mundo rural, visto a través de algunas interacciones del modelo residencial dentro del marco de la conurbación del desplazamiento laboral. En el valle de Mexicali, la agricultura es aún la actividad principal del espacio rural, pero sus objetivos y sus medios se han modificado. Este espacio ofrece un campo de visión de primer orden para el análisis de la relación con el complejo urbano industrial. Se resalta aquí la idea de la participación a distancia y de la reconfiguración aureolar dentro de una ecuación que suma funcionalidad y subordinación espacial, integración territorial y polarización de oportunidades del desarrollo.
\end{abstract}

\begin{abstract}
Do we have the means to make two politics, the urban and the rural? Can we consider the salvation of a rural world in another alternative different to that of the agriculture politics? In this research, more than contributing solutions, diverse questionings are made; the purpose of this is to reflect on the debate of the space mutations that affect the rural world, seen through some interactions of the residential model within the conurbation of the labor breakout. In the Mexicali Valley, agriculture maintains itself as the main activity in the rural environment, but its objectives and its means have modified. This environment offers a first hand vision for the analysis on the relationship with the complex urban industry. Here, the idea of the distance participation and space subordination, territorial integration and opportunity polarization for development is highligted.
\end{abstract}

Actualmente, en lo que respecta al espacio rural, la investigacion se ha hecho más concreta. Las mutaciones territoriales y las recomposiciones espaciales son tales, que se impone buscar nuevos enfoques más audaces $y$, sobre todo, realizar una renovación de los conceptos.

* Investigador del Instituto de Investigaciones Sociales de la Universidad Autónoma de Baja California. E-mail: toudert@faro.ens.uabc.mx

N.de E. Traducido del francés por Danielle Wynants.

Este artículo es fruto de las discusiones efectuadas separadamente con mis colegas Arturo Ranfla y José A. Moreno. Asumo, sin embargo, la responsabilidad del análisis desarrollado. 
En la conciencia colectiva, aún se perciben los modelos residenciales bajo una optica de espacio jerarquizado y sumisa a las nociones de proximidad, de la difusión de las ideas y de los actos en relación con los factores de pluralidad de las escalas temporales (resistencia, fragilidad, competencias, etcétera). Globalmente, estamos todavia bajo el dominio de la alteridad espacial. ${ }^{2}$ ¿No podriamos considerarnos de una manera global como actores de la relación de fuerza de los grupos sociales implicados en el uso de los territorios y del significado que ellos les atribuyen?

Georges Bertrand (1992), ${ }^{3}$ pone en relación causal funcionalidad y durabilidad del paisaje. Para él, un paisaje no subsiste a menos que sea funcional. Para Larcena, et. al. (1996), la funcionalidad de un paisaje rural está relacionada en parte con la evolución sectorial, tratándose aquí de una producción pasiva del espacio rural, la otra parte la contiene el volumen de la intervención del poder público para regular las dinámicas del paisaje: es la producción activa. ¿No es el peso de la producción activa el reflejo de la importancia social asignada a un grupo humano localizado?

Para los países industrializados, si el hecho de residir en una zona rural no significa una gran diferencia estatutaria respecto a sus conciudadanos urbanos, para los países en vías de desarrollo, el dualismo urbano/rural queda aún como un dato intrínseco a las estrategias de desarrollo y del ordenamiento territorial. Los cuestionamientos de la integración social y espacial nunca han sido tan vivos como los son hoy. La esterilidad del debate sobre el volumen de la produccion activa, aparte de su contenido ideológico, ha demostrado su incapacidad "genética" de ir más lejos de las soluciones tradicionales. En el momento donde florecen las necesidades de un reparto a todos los niveles, ¿podemos bablar de un reparto de oportunidades dentro de las permutaciones eventuales de las redes reticulares existentes o las que se están formando? La pregunta gravita alrededor "de la continuidad en la ruptura", una evolución dialéctica de los grandes conjuntos territoriales al ritmo de las recombinaciones de naturalezas múltiples. La práctica de la geografía de las redes confirma esta hipótesis para los grandes conjuntos nacionales y regionales, y para la escala local, ¿cómo se comportan las agrupaciones humanas frente a los cambios estructurales?, ¿existen factores que estructuran las mutaciones espaciales?

En este trabajo, trataremos de analizar la distribución espacial de la población rural en el valle de Mexicali según información del Censo de

2 En el sentido de la confrontación de Ios perfiles sociopsicológicos identificados por un típo de organización espacial diferente, si no es que contradictoria.

En la introducción al coloquio "Le Patrimoine et Enjeu de Developpment. Francia", 1992. 
población y vivienda ejidal (ver figura 1). Dentro de los límites de los datos estadísticos y gráficos de los cuales disponemos, ${ }^{4}$ nos haremos camino para demostrar, por una parte, las lógicas de organización de las agrupaciones humanas y sus numerosas conexiones con la ciudad de Mexicali, y por otra, el sistema de las ciudades vecinas. En segundo lugar, nos parece necesario resaltar las características laborales y residenciales. ${ }^{5}$ Para finalizar, concluiremos este trabajo con la articulacion de estas dos nociones dentro del nuevo sistema complejo del "paisaje compartido".

Antes de empezar este estudio, es indispensable definir la problemática en el contexto espacio-tiempo evolutivo, pues el valle de Mexicali actual no es ciertamente un producto de la casualidad.

\section{DEL DESIERTO BROTA EL OASIS: CRÓNICA DE UN ESPACIO TOTALMENTE ORDENADO}

Contrario a lo que generalmente se admite, hacer el inventario de los factores que precedieron el nacimiento del valle de Mexicali no es tan complejo. En efecto, podemos eliminar de golpe todos los elementos de la historia anteriores a un siglo y hacer caso omiso de los factores naturales para explicar los diferentes paisajes generados (Portais, 1995:275-287).

El valle de Mexicali es una obra totalmente reciente, la cual surge a principios de este siglo, con la desviación del río Colorado para el riego de la franja noroeste estadounidense del bajo delta del mismo río. La valoración del oasis de Mexicali, arranca casi al mismo tiempo con los esfuerzos realizados para su continuidad estadounidense (Anguiano, 1995), apoyándose en la inversión extranjera (Ranfla, 1991:131-144), hecha realidad después de adoptar una dialéctica de desarrollo sobre los territorios fronterizos a través de las concesiones territoriales (Moreno Mena, 1994).

4 Consultar la información sobre las localidades en el Censo de población y vivienda, 1990. resulta difícil debido a que los nombres mencionados no coinciden con los presentes en el mapa topográfico del INEG (1972). Igualmente, la georeferenciación que viene con estas localidades no es correcta. Estas dificultades de uso nos llevaron a escojer el Censo de población y vivienda ejidal, 1990, que a través de la localización del poligono ejidal permite la ubicación de las localidades rurales en el valle de Mexicali. Esta metodología de trabajo se utilizó para todas aquellas localidades de menos de 2500 habitantes. Para las de mayor población se usóel Censo de población y vivienda, 1990. Asimismo, es pertinente aclarar que los poblados ubicados dentro de las colonias que conforman el valle de Mexicali se excluyeron de nuestro análisis por las razones antes expuestas.

En este caso, en característica residencial queremos trazar algunas líneas que definen al residente como un agente complejo dentro de la comunidad, con la que comparte, además. algunas manifestaciones del espacio vivo y del espacio vivido. 


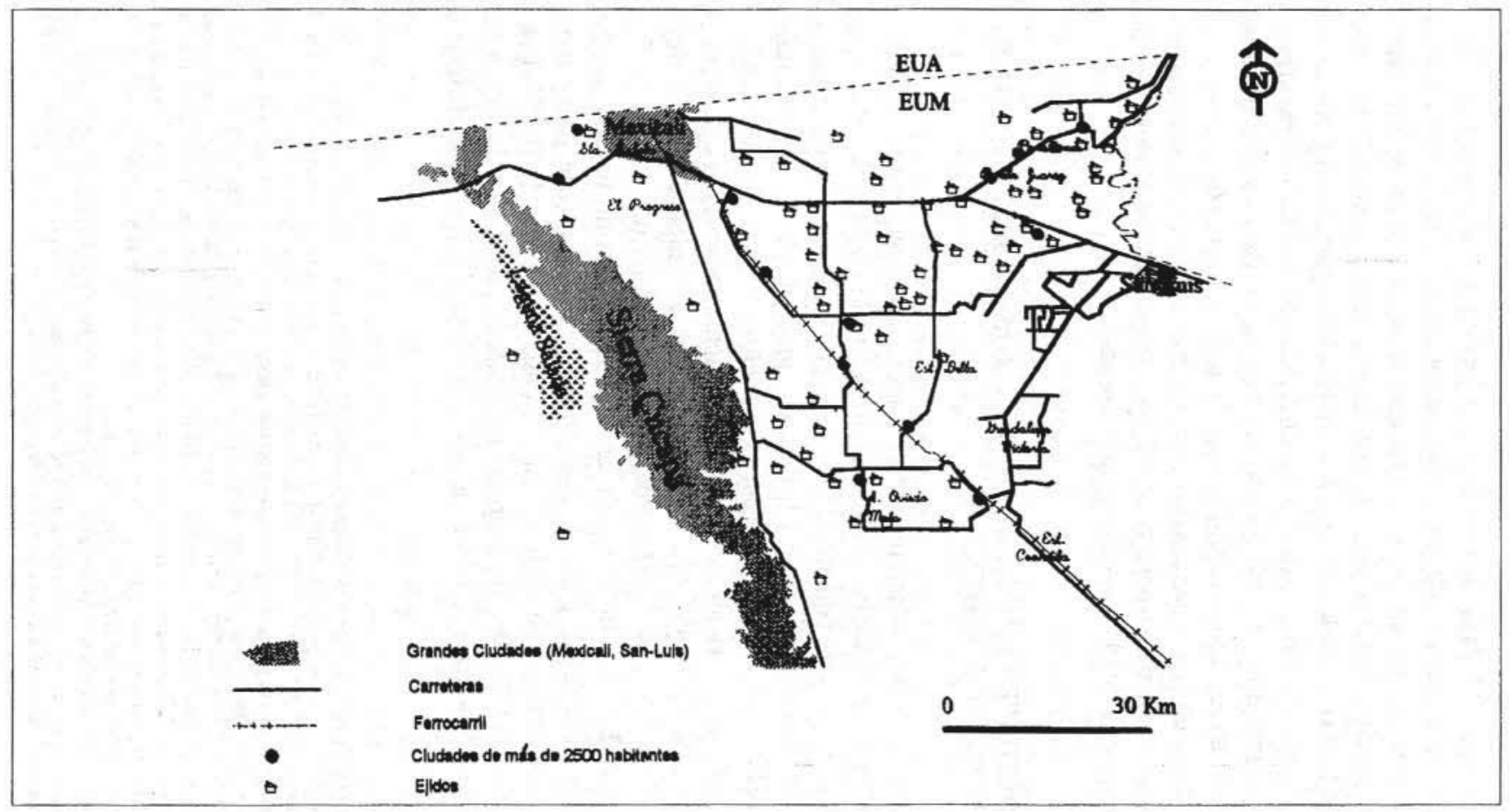

Figura 1. Ubicación y situación geográfica. 
El frente pionero ${ }^{6}$ asociado al capital extranjero, produjo en el valle su primer algodón en 1912 (Ranfla, 1991:132), pero su gran mérito consistió en la implantación de un oasis y las primicias de una infraestructura agricola en pleno desierto. El desarrollo progresivo de esa nueva región rápidamente fue confrontado con el factor bumano limitante (Moreno Mena, 1994:166). El flujo migratorio se convirtió en factor determinante para la continuación y la extensión de la actividad agrícola, así como de la configuración de la dimensión humana y cultural de la identidad cachanilla (Toudert, 1995a). En efecto, el crecimiento de la población entre 1900 y 1910 fue de aproximadamente $400 \%$; la de los diez años siguientes fue el doble. El crecimiento de esa franja en Baja California fue tal que, en 1915, Mexicali ascendió al estatuto de municipio, y un poco más tarde, superó aun a Ensenada, convirtiéndose en la capital del Territorio Norte de la Baja California, marcando, de esta manera, el fin del predominio del eje de desarrollo sur-norte, y poniendo énfasis en el papel del poder espacial fronterizo como punto de intersección de las condiciones objetivas y subjetivas para la emancipación de un capitalismo de tipo agrario.

De acuerdo con el Censo de población de 1921 (cit. en Anguiano, 1995), se confirma una organización espacial y funcional de proximidad que define aquí el hábitat rural con el lugar de trabajo y los ejes de comunicación, en el cual el ferrocarril intercaliforniano figura ya como el primer eje de desarrollo residencial del valle de Mexicali (Ranfla, 1991:134). En ese mismo periodo, es muy interesante mencionar la fuerte dispersión y la débil densidad de los poblados,' exceptuando las ciudades de Mexicali y Los Algodones (ver cuadro 1).

La alianza con el capital extranjero en la región fue, sin duda, uno de los factores primordiales del desarrollo local y regional, pero en la buella del éxito económico, esa asociación produjo también una fuerte contradicción social. En efecto, la concentración del capital y de los medios de producción eran de tal forma que podían causar problemas algún día. ${ }^{8}$

La solución del conflicto distribuidor se llevó a cabo continuamente bajo la presión de tres factores, en función de su importancia y del peso de la intervencion del gobierno central:

- El flujo migratorio hacia la región aumentó con el deterioro del mercado de trabajo del otro lado de la frontera, como fue el caso de

6. Según una expresión del geógrafo frances Pierre Monbieg.

7 Lapoblación del municipio de Mexical ien 1921 era de 14599 habitantes, que representaba ya $62 \%$ de la población total del territorio norte de Baja California.

s La Colorado River Land Company (CRLC) monopolizaba prácticamente la totalidad de las tierras de cultivo y los servicios a la agricultura. En 1930, ia CRLC era oficialmente propietaria de 323688 ha. 


\section{CUADRO 1. Evolución diacrónica de la distribución de los poblados y de su razón social (1921-1950).}

\begin{tabular}{lrrrr}
\hline Agrupaciones & 1921 & 1930 & 1940 & 1950 \\
Pueblos & 2 & 3 & 3 & 2 \\
Colonias agrícolas & 1 & 18 & 54 & 73 \\
Rancherias & 105 & 1 & 7 & 5 \\
Ranchos & 27 & 11 & 11 & 7 \\
Ejidos & - & - & 65 & 65 \\
Campos agrícolas & - & 14 & 2 & 1 \\
Estaciones de fertocarril & 14 & 14 & 18 & 11 \\
Hacienda & - & 1 & - & - \\
Otros & 3 & 2 & 3 & 1 \\
Presa & - & - & - & 1 \\
Total & 152 & 64 & 163 & 166 \\
\hline
\end{tabular}

FUENTE: María Eugenia Anguiano (1995:128).

la crisis de 1929, que hizo girar la "válvula de escape" de retorno hacia las zonas fronterizas.

- Lamaduración y la afirmación de la clase empresarial en los poderes local y regional, como actores que se afirman primero como nacionales, apresurados por terminar con la asfixia monopolística extranjera que los integra poco o muy mal a las nuevas vocaciones económicas, y redujo de manera considerable su poder de ensanchar sus bases de clientela.

- La fusión del poder central con "las reivindicaciones populares", tendencia que se reafirmó con más fuerza en el sexenio de Lázaro Cárdenas.

La adopción de la reforma agraria y la apertura de la región como zona libre, constituyeron, junto con los grandes esfuerzos del desarrollo del oasis, los nuevos fundamentos de un desarrollo sostenido. El paisaje rural, entre otros, cambió rápidamente de una frontera borrada a un conjunto de redes marcado por lógicas territoriales reales y fuertes tendencias de impresión de la identidad hacia el espacio (Portais, 1995:276). Ese periodo presentó grandes trastornos en el modelo residencial en el valle de Mexicali. El acceso a la tierra de numerosos colonos inauguró la razón social residencial o sencillamente la noción del reagrupamiento colectivo laboral. Esa manera de organizar el hábitat rural cumplía dos objetivos: por una parte, el de la proximidad y de la maximización del control sobre los 
nuevos recursos adquiridos; y por otra, un esquema de traslación de la razón jurídico-económica hacia una legitimación social y una subordinación del beneficiario y de su familia a un orden político-sindical que se desdibujaba en la región. En función de la distribución de la tierra, se constituyeron ejidos y colonias en el valle y se consolidaron reagrupamientos residenciales con base laboral; otros nacieron de ese nuevo orden, sin embargo, algunos perdieron su importancia e incluso desaparecieron. Fuera de esa nueva organización del espacio, el grupo de poblados que emergí con el ferrocarril Intercalifomiano extendió sus límites hacia el sur, saliendo completamente de su área de influencia. La puesta en marcha del ferrocarril Sonora-Baja California permitio el establecimiento de cierto número de asentamientos humanos, como las ciudades-estaciones y el nacimiento de una nueva área de crecimiento residencial. La pérdida de interés hacia el ferrocarril Intercaliforniano y su sustitución por la carretera Mexicali-San Luis con el tramo Santa Rosa-Los Algodones, y la construcción de las carreteras Mexicali-Tecate y Mexicali-San Felipe, finalizaron el modelo residencial rural de Mexicali alrededor de tres áreas de concentración (Ranfla, 1991:134-135): a) la ciudad de Mexicali y su zona suburbana inmediata; b) la zona noroeste, alrededor de Ciudad Morelos, y c) la zona sur, alrededor de Ciudad Guadalupe Victoria y Estación Coahuila (ver figura 1).

El reparto espacial de las ciudades ${ }^{9}$ y poblados en el valle de Mexicali, se detuvo e incluso bajo en población casi al mismo tiempo que cuando el ciclo del algodón llegó a su fin como motor de desarrollo, y a la vez que se puso en marcha el ciclo maquilador que movilizo, sobre todo, los recursos urbanos. La poblacion de la ciudad de Mexicali que representaba $52 \%$ de habitantes del municipio en 1940 , pasó a $67 \%$ en 1980 y $73 \%$ en 1990 (INEGI, 1992). Mexicali, que aseguraba la existencia para las localidades rurales, se volvió rápidamente un "ogro urbano", devorando los poblados del antiguo cinturón (Collin y Toudert, 1995).

En la zona rural, ni el peso de la producción pasiva desempañada a través de los programas de la federación (Ranfla, 1991:136), ni el desarrollo de los cultivos de hortalizas (Stamatis, 1993), pudieron contrarrestar la tendencia urbana industrial. Menos los precios de garantía y después el Procampo a través del arranque del ciclo del trigo (Toudert, 1995b), fueron capaces de ofrecer un desarrollo continuo, como lo proporciono el algodón durante más de medio siglo. Las diversas mutaciones en el espacio económico regional, nacional e internacional en la esfera de los

9 Según la clasificación del INEGl, que define la ciudad como un agrupamiento de más de 2500 habitantes. 
intercambios comerciales donde se localizan los sectores motores, se han desarrollado no forzosamente en contra del sector primario, sino en contra del ideal de integración, que puede generar la agricultura hasta en sus intimos rincones de su mercado laboral. De allísu incapacidad para generar o mantener un modelo residencial competitivo (en calidad y en cantidad) con su homólogo urbano.

\section{RURAL, RURALISMO Y RURALIDAD DE TIERRAS DENTRO DE LA CONURBACIÓN: LAS MUTACIONES DE LAS VOCACIO- NES DEL ESPACIO}

Los cambios del modelo de consumo internacional han generado una reconfiguración de la demanda y su segmentación. La competencia por los mercados se ha vuelto feroz y la adaptación de los centros de producción agrícola no se ha hecho totalmente a través de una adaptación natural de todas las condiciones, sino sumando todas las ventajas comparadas en el proceso de la producción, ya sea minimizando los costos de transformación de las explotaciones o sencillamente cediendo la actividad a actores más experimentados en ese género de transacciones (Toudert, 1995b:61). La naturaleza de las condiciones de producción, la saturación del mercado intemacional tradicional y la complejidad de los factores limitantes naturales, hacen que la producción de las hortalizas de Mexicali sea menos diversificada ${ }^{10}$ e incapaz de crecer continuamente al mismo ritmo de los años ochenta (Toudert, 1995a). La producción de granos se expandió con el Procampo para dominar al patrón de cultivo (ver cuadro 2 ), pero es todavía precaria, incluso si recibe más apoyo. ${ }^{11}$ Los granos, y particularmente el trigo, por el hecho de la gran mecanizacion, ofrece cada vez menos trabajo, ${ }^{12}$ pero de manera paradójica recibe cada vez más subsidios. $^{13}$

${ }^{10}$ J.P. Hernández apunta que durante un año normal son cultivadas en el valle 10000 ha de hortalizas con 4000 ha de cebollines y 3000 ha de espárragos; las otras 3000 ha tienen 15 cultivos más. La concentración de la superficie del patrón de cultivo de hortalizas para "las producciones pivote" como el espárrago, cebollin y rábano, hacen que la producción de hortalizas sea menos diversificada y vulnerable (A campo, febrero, 1997).

${ }_{11} \mathrm{La}$ deuda de los trigueros y sus demandas por un mejor precio de venta ha sido uno de los problemas de la Secretaría de Agricultura más importantes de los últimos años.

Según las cifras de la delegación de Baja California de la SARH, el rendimiento de la fuerza total de trabajo necesaria para el cultivo de una hectárea de trigo sobre la misma fuerza para el espárrago es de 0.05 .

13 El importe de Procampo se aproxima a los 4.5 millones de nuevos pesos para el ciclo primavera-verano 1996 con 1168 solicitudes y una superficie de 9000 ha; para el ciclo otoño-invierno 1995-1996, el mismo programa desembolsó una suma de 44000000 de pesas. Para 1997, un aumento de 10\% a la prima por hectárea de los cultivos cubiertos por Procampo es previsto desde ahora. 


\section{CUADRO 2. El padrón de cultivo del distrito de riego 014 para el ciclo primavera-verano 1996/1996.}

\begin{tabular}{lrc}
\hline Cultivos & Total sembrado (ha) & Proporciones (\%) \\
\hline Trigo & 66956 & 82.90 \\
Cártamo & 1023 & 1.26 \\
Cebada & 266 & 0.32 \\
Rye-grass & 5072 & 6.28 \\
Cebollin $_{\text {Varios }}{ }^{\mathrm{a}}$ & 3914 & 4.84 \\
Total & 3524 & 4.36 \\
\hline
\end{tabular}

FUENTE: SAGAR, delegación estatal de Baja California, Distrito de riego 014, nío Colorado, Mexicali, 1997.

a La producción agrícola del valle de Mexicali opera en dos ciclos agrícolas: oton̄o-invierno y primavera-verano. Después del fin del ciclo del algodón y las reducciones drásticas en los volúmenes de agua, el ciclo primavera-verano, que normalmente debe recibir la reconversión hortalicera, no fue totalmente realizada. En este sentido, el volumen de la producción de hortaliza para el primer ciclo es muy importante dentro de la producción total, y en términos de la expansión, sin tomar en cuenta el cebollín, notamos que la superficie hortalicera (englobada dentro del puesto varios) es muy baja frente a los cereales de consumo humano.

La dificultad de encontrar otras salidas sobre el mercado internacional y la reducción del poder adquisitivo interno, repercute sobre todos los componentes del desarrollo. La reducción del espacio rural clásico, que ya es un hecho consumado, ${ }^{14}$ no está totalmente relacionado con las múltiples revisiones de los términos del intercambio del sector agrícola, tiene mucho que ver con el crecimiento de la actividad económica de Mexicali (la ciudad y su alrededor) y la actualización de la segregación salarial por la fortificación del reglamento social en el dualismo urbano/rural.

El sector primario en la población económicamente activa (PEA) del municipio no ha dejado de bajar: de $61.6 \%$ en 1950, descendió en 1980 a $26.5 \%$ y $22.3 \%$ en 1990 (INEGI, 1994). Esas cifras muestran una polarización de la actividad económica esencialmente alrededor de la industria y de los servicios. La empresa maquiladora pasa de 54 unidades en 1982 a 124 en 1994; el personal ocupado por el mismo periodo está multiplicado por 3.42. Estas modificaciones en la estructura del trabajo han llegado incansablemente a reducir la participación del sector primario en la formación del PIB: esa participación pasa de $46.6 \%$ en 1950 a $16.1 \%$ en 1980 , demostrando la pérdida del dinamismo frente a otros sectores de actividad

${ }^{14}$ Ese sector empieza ya a mostrar señales de agotamiento a partir de los años sesenta. 
(Agenda estadistica de municipio de Mexicali, Baja California, 1990). Para un espacio rural donde la principal actividad reside todavía en la agricultura, esas cifras son las de un espacio "bajo perfusión" (ver cuadro $3),{ }^{15}$ Es todo un conjunto de ciudades y poblados que se encuentran, por así decirlo, prisioneros del concepto de la vocación del espacio cada vez más en conflicio (Toudert, 1997). La competencia por los recursos no beneficia a las zonas rurales tradicionales, el desequilibrio entre las percepciones y las deducciones no puede ser indefinidamente ignorado y la democratización continua de la sociedad municipal en ausencia de una política de ordenamiento del territorio bajo una óptica de integración dinámica y de solidaridad, tiene el riesgo de generar más que un conflicto distribuidor, un filtrado espacial. En una situación de falta de recursos públicos y una inflación de la demanda residencial urbana se puede arriesgar a que se imponga sencillamente una "solución política" a los problemas del desarrollo en beneficio de las zonas con fuertes concentraciones humanas.

CUADRO 3. El balance entre los ingresos y los egresos públicos para algunas ciudades pequeñas del valle de Mexicali (en miles de pesos, 1980=base).

\begin{tabular}{lrr}
\hline Localidades & 1988 & 1989 \\
\hline Batáquez & -669 & -636 \\
Benito Juárez & -683 & -602 \\
Cerro Pricto & -708 & -605 \\
Ciudad Morelos & -338 & -280 \\
Colonias Nuevas & -70 & -96 \\
Estación Delta & -296 & -307 \\
González Ortega & -55 & 179 \\
Vicente Guerrero & -175 & -186 \\
Guadalupe Victoria & -312 & -310 \\
Hermosillo & -558 & -551 \\
Hechicera & -638 & -2 \\
Progreso & -78 & -41 \\
Venustiano Carranza & -46 & 100 \\
San Felipe & 565 & 1429 \\
\hline
\end{tabular}

FUENTE: Agenda estadistica delmunicipio de Mexicali, Baja Califomia(1990:111-112).

${ }^{15}$ En el sentido que el mantenimiento y desarrollo del espacio rural está sometido al presupuesto del Estado. 
Al concepto de ruralidad se le golpeó de fuerte manera. ¿Qué significa actualmente el ruralismo o sencillamente la palabra "rural" en el valle de Mexicali? El ruralismo que por mucho tiempo fue confundido aquí con el agrarismo, finalmente no tuvo el tiempo ni la estructura necesarios para generar un bosquejo de cultura y de identidad profunda y bomogénea. El flujo migratorio que abarca esa región a partir de los estados que expulsan población (ver cuadro 4) (Schmidt, 1994), lleg6 sobre todo a confirmar el papel del espacio integrador durante y justo después de la reforma agraria. Ese espacio providencia se redujo con el principio y el recrudecimiento del conflicto sobre los recursos después de la internupción del reparto. $\mathrm{La}$ modificación de los términos del intercambio dio el golpe de gracia al modelo de integración rural ${ }^{16}$ por la actividad agrícola. ¿Las mutaciones espacio-productivas conllevan al fin de la ruralidad en el valle de Mexicali, o sencillamente al fin del modelo rural tradicional ? $^{17}$

La población rural municipal pasó de $21.16 \%$ en 1980 a $12.5 \%$ de la población total en 1993 (Agenda estadística del municipio de Mexicali, Baja California, 1990 y IV Informe de gobierno, 1993, respectivamente). La labor agrícola en general no concierne más que a la mitad de la población económicamente activa (PEA) ejidal (INEGI, 1991). El examen de las estadísticas disponibles muestra que globalmente la polarización en el sector primario está de manera inversa relacionada con la urbanización y la concentración de la población (ver figura 2), pero parecería que el

\section{CUADRO 4. Evolución de la parte de la población no nativa dentro de la población total de Mexicali.}

\begin{tabular}{ccc}
\hline Años & Población nativa(\%) & Población no nativa(\%) \\
\hline 1940 & 37 & 63 \\
1950 & 35 & 65 \\
1960 & 36.6 & 63.4 \\
1970 & 64.5 & 35.5 \\
1980 & 60.8 & 39.2 \\
1990 & 63.3 & 36.7 \\
\hline
\end{tabular}

FUENTE: Censos de población y vivienda respectivos a los años mencionados.

\footnotetext{
${ }^{16}$ En términos de la existencia de una identidad espacial funcional auténtica capaz de mantener un proceso de integración duradero dentro de una macroestructura (por ejemplo la región o el estado).

${ }_{17} \mathrm{Para}$ el modelo rural tradicional se entiende una relación de ruptura con otros modelos de integración territorial vigentes.
} 


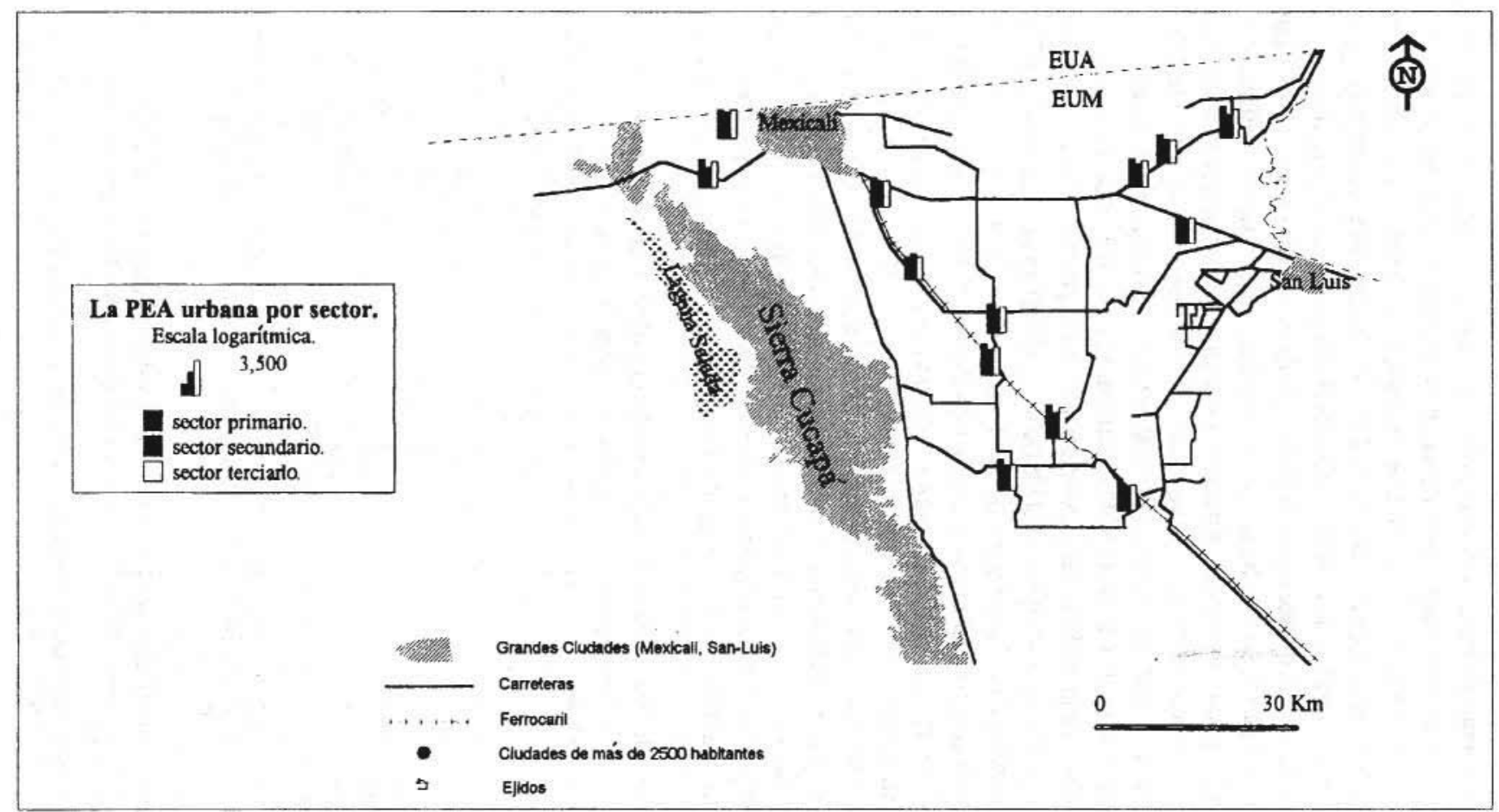

Figura 2. Distribución de la PEA de los poblados de más de 2500 habitantes por sector de actividad. 
reparto por sectores de la PEA es generalmente diferente en función del tipo de localidades (ver cuadro 5) y distancia que las separa de las grandes ciudades como Mexicali y San Luis Río Colorado, Sonora. En efecto, para la poblacion ejidal, el vecindario de las grandes ciudades hace que aumente la parte del sector secundario y terciario en el PEA, lo que nos hace pensar en "ejidos dormitorios". La proximidad a los ejes de gran tráfico tienden a favorecer una sectorización laboral mucho más diferente del esquema tradicional (ver figura 3). ${ }^{18}$ La distancia como elemento regulador de la nueva sectorización laboral, aparece de manera más clara en el análisis de las ciudades pequeñas de más de 2500 babitantes. ${ }^{19} \mathrm{La}$ distancia que separa esas ciudades de los grandes centro urbanos de correspondencia (la ciudad de Mexicali o la de San Luis Río Colorado), muestra de manera muy clara la disminución relativa del sector primario en función de la reducción del trayecto al centro urbano de correspondencia. ${ }^{20}$ Esa relacion más allá de la primera lectura que puede hacerse en el sentido de la confirmación del desplazamiento laboral cotidiano de una franja de la población activa, confirma también un efecto de polarización alrededor de las grandes ciudades de la región. ${ }^{21}$

\section{CUADRO 5. El reparto sectorial de la PEA en arreglo a los tipos de localidades.}

\begin{tabular}{lcccc}
\hline $\begin{array}{l}\text { Tipos de } \\
\text { localidades }\end{array}$ & $\begin{array}{c}\text { Número } \\
\text { de ciudades }\end{array}$ & $\begin{array}{c}\text { Sector I } \\
(\%)\end{array}$ & $\begin{array}{c}\text { Sector II } \\
(\%)\end{array}$ & $\begin{array}{c}\text { Sector III } \\
(\%)\end{array}$ \\
\hline $\begin{array}{l}\text { Ejidales }^{\mathrm{a}} \\
\begin{array}{l}\text { Ciudades de más de } \\
2500 \text { habitantes }^{\mathrm{b}}\end{array}\end{array}$ & 74 & 59.53 & 18.69 & 21.09 \\
\begin{tabular}{l} 
Mexicali (ciudad) \\
\hline
\end{tabular} & 14 & 40.83 & 18.46 & 40.69 \\
\hline
\end{tabular}

FUENTE: XI censo general de población y vivienda, INEGI, 1990.

${ }^{a}$ El total de los 71 ejidos, 2 NCFE y 1 NCPA del Censo general de población y vivienda ejidal, 1990.

${ }^{\mathrm{b}}$ Sin la ciudad de Méxicali.

${ }^{18}$ Entendemos por esquema tradicional a la polarización de la ocupación laboral alrededor del trabajo agricola.

19 Consideradas en el sistema nacional de clasificación de agrupaciones humanas como base mínima para la organización de una comunidad urbana.

${ }^{20}$ La noción de centros urbanos de correspondencia es empleada aquí para definir las áreas de influencia a partir de esos centros que incluyen las ciudades de más de 2500 babitantes y los otros poblados de menor importancia residencial.

${ }^{21}$ La noción de región aqui es empleada para designar el valle de Mexicali en su totalidad, rebasando sobre el municipio de San Luis Río Colorado. 


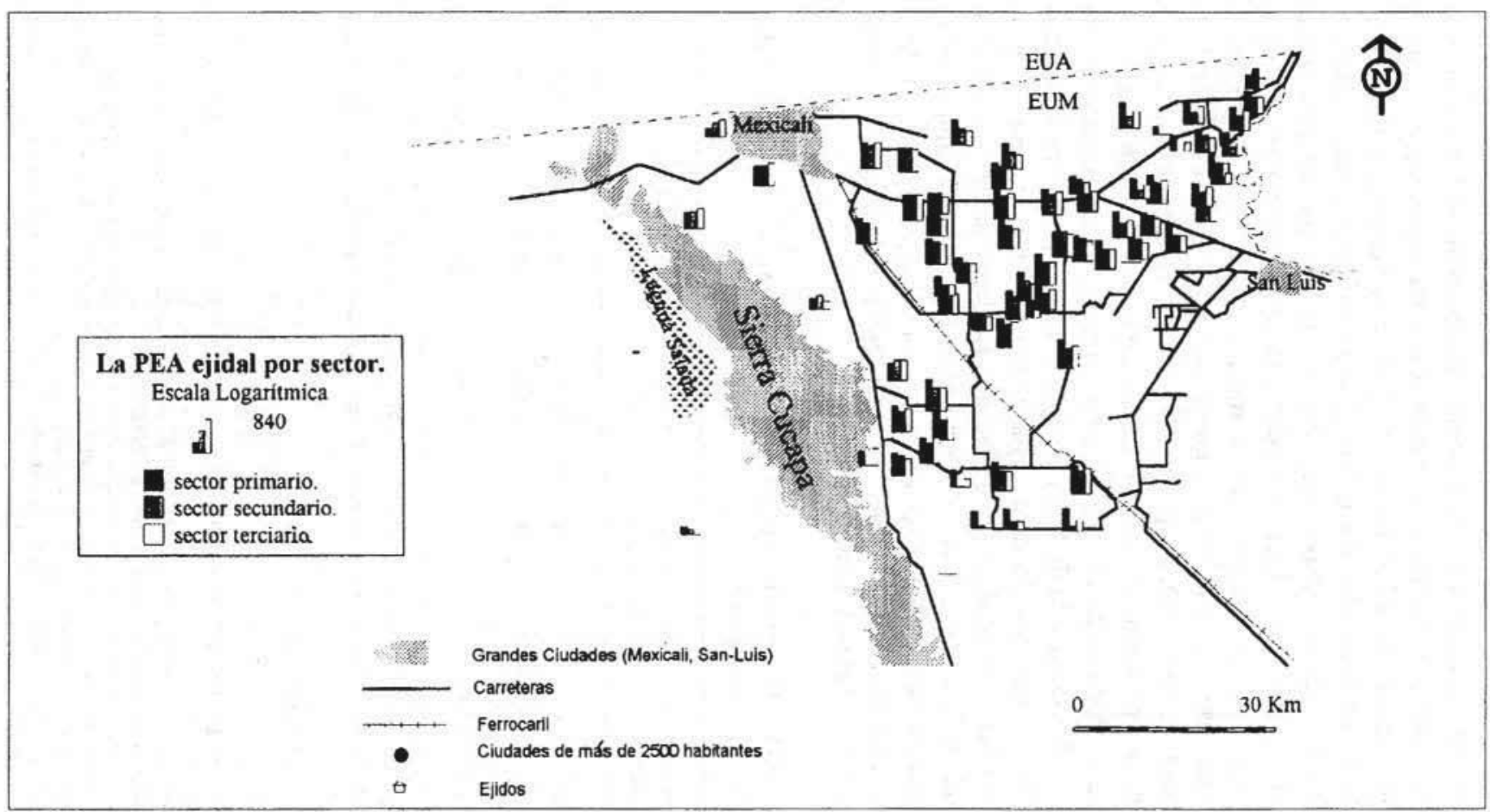

Figura 3. Distribución de la PEA ejidal por sector de actividad. 
Una vez más, esa polarización puede salir del marco de los límites municipales definiendo áreas de influencias intermunicipales. El análisis de las áreas de influencia, muestra la articulación de la arquitectura de la red portadora del conjunto residencial del valle de Mexicali. De una manera global, constatamos que está organizado alrededor de dos niveles de polarización jerárquicas: el primer nivel está constituido por un sistema real de conurbación que pone en juego la conexión de los grandes centros de concentración humana, con el panel de las ciudades de más de 2500 habitantes: "las ciudades pequeñas"; el segundo nivel, en cambio, pone en juego la relación espacial de los ejidos con "las ciudades pequeñas". La articulación de los ejes define, pues, un sistema de interdependencias jerárquicas organizadas en un conjunto regional de redes cuyas dos variables determinantes parecen gobernar la ecuación residencial: el trayecto a los centros motores y la concentración de la población. ${ }^{22}$

La noción de la red reticular resalta mejor si restituimos la espacialidad de los poblados ejidales en el interior del sistema de "las ciudades pequeñas"; en efecto, el examen de la figura 5 muestra que la nube ejidal está constituida por cinco zonas y una sexta compartida:

1. La zona periurbana de Mexicali (Z1): Constituida por la red matriz Mexicalí y sus satélites de más de 2500 habitantes. Es un conjunto restringido de ejidos dispersos. El predominio de la nueva polarización sectorial, la disponibilidad de los terrenos urbanos y el reparto de los servicios en arreglo a la concentración han caracterizado a esa zona casi urbana.

2. La zona de San Luis Rio Colorado (Z2): Esta zona gira alrededor de la ciudad de San Luis Río Colorado y tres ciudades pequeñas. Cerca de ese dispositivo urbano se coloca un grupo de ejidos sobre el costado este. El sector primario de esa zona, al contrario de la Z1, es aún importante, pero constatamos una tendencia progresiva al sector terciario dinamizado a partir de la frontera norte, la industria, los servicios de la carretera Mexicali-San Luis Río Colorado y la ciudad de San Luis Río Colorado.

3. La zona de Morelos (Z3): Es un complejo denso de ejidos que giran alrededor de dos ciudades de más de 2500 habitantes: Ciudad Victoria y Ciudad Morelos. La proximidad de la frontera proporciona un nivel interesante de empleos en la industria y los servicios, pero aquí la agricultura domina todavía el sector laboral.

\footnotetext{
22 Seria, sin embargo, muy arriesgado creerse implicado de una manera sencilla en el análisis clásico de gravedad, ya que si la interacción de los modelos residenciales obedece aquí a factores que se pueden medir, está probablemente relacionada también con elementos subjetivos cuya influencia, a lo que menos se puede decir aquí, no ha sido aproximada por la investigación aún.
} 


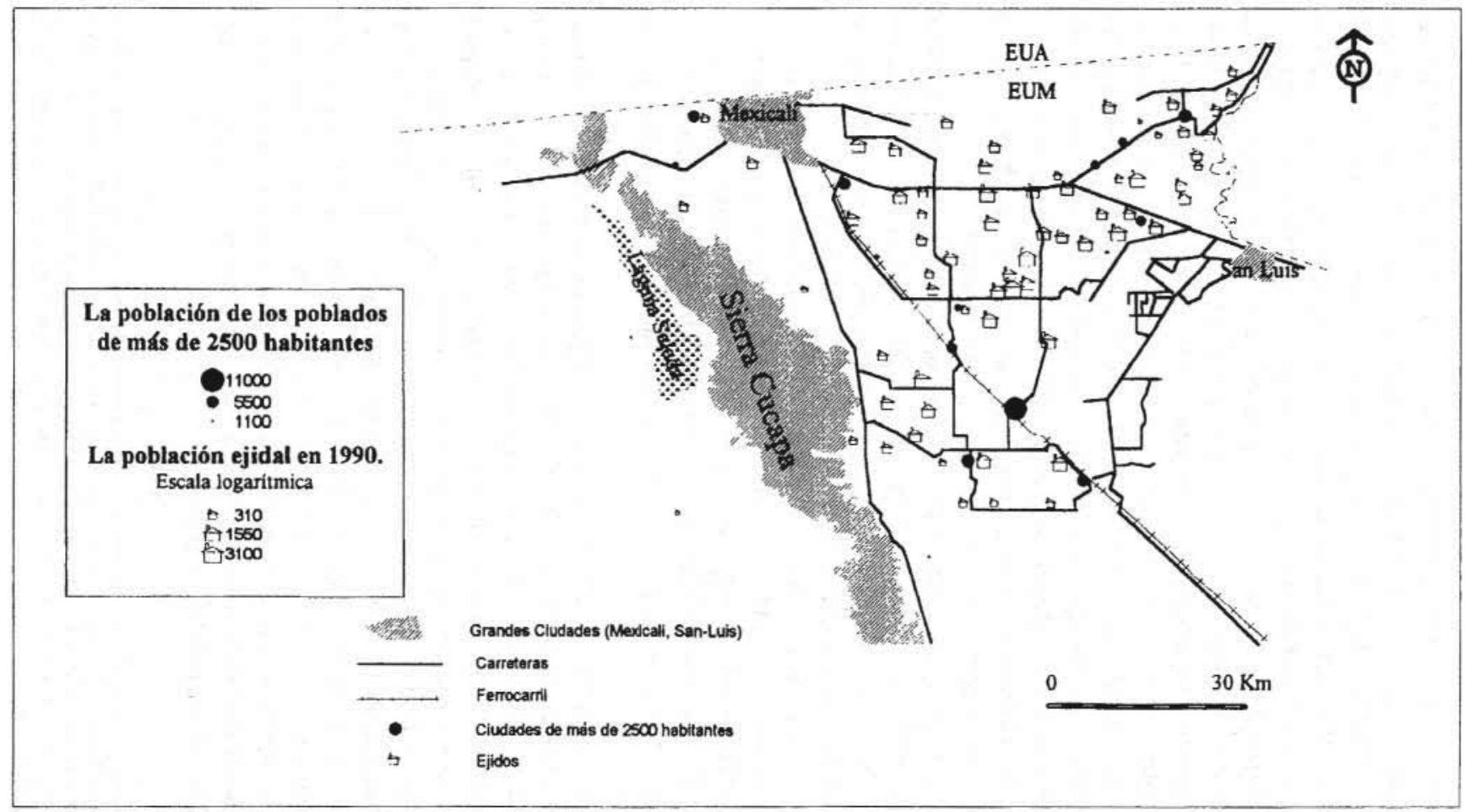

Figura 4. Distribución espacial de la población. 
4. Estación Coahuila (Z4): Constituida por tres ciudades pequeñas de más de 2500 habitantes y un importante grupo de ejidos. El sector primario se hace cada vez más importante hacia el sur; las otras ocupaciones laborales se fortalecen más en la cercanía de la carretera Mexicali-San Felipe.

5. La zona Estación Delta (Z5): Esta zona está constituida alrededor de las dos estaciones de ferrocarril: Delta y Victoria. Ambas representan un peso de más de 2500 habitantes. Aquí, el sector primario es aún mayoritario, la proximidad de la carretera ${ }^{23}$ facilitó la transportación cotidiana de los trabajadores para la industria y los servicios hacia la ciudad de Mexicali.

6. La gran zona compartida (Z6): Esta zona agrupa a los ejidos colocados en el centro-norte del valle de Mexicali; los ejidos periféricos de las zonas antes citadas también pueden formar parte de esa agrupación que no tiene la capacidad de generar ciudad alguna de más de 2500 habitantes. ${ }^{24}$ En su totalidad, esta zona permanece dominada por su perfil agrícola, pero presenta una exportación de mano de obra hacia los otros sectores, principalmente el secundario. Más que un espacio compartido. esta agrupación constituye una zona de transición en el interior de un gran espacio de discontinuidad residencial.

El reparto de población (figura 4) en las ciudades pequeñas de más de 2500 babitantes obedece a una lógica de concentración para las ciudades estaciones de ferrocarril (ciudades dormitorios). Para las otras ciudades pequeñas de más de 2500 habitantes es generalmente homogénea, no obstante, con un repunte importante en las cercanías con los grandes centros de actividad (ver cuadro 6).

Para los ejidos, el reparto de la población sí responde a la noción de una concentración más grande en las cercanías de los ejes de circulación, y no constituye una regla general para el conjunto de las estructuras de los poblados ejidales. La ciudad pequeña de más de 2500 babitantes ejerce un efecto inhibidor sobre el nivel de concentración humana ejidal.

La red de carreteras en la estructuración de la vivienda rural en Mexicali es, sin duda, un elemento predominante en el seguimiento de la competitividad del modelo residencial rural, y eso a pesar de la crisis laboral del sector agrícola. El alto índice de la presencia del automóvil en la vida cotidiana y su contribución en reducir el espacio vivo, constituye, para el municipio de Mexicali, el otro factor que permite la rapidez y la

${ }^{23}$ El ferrocarril ya ha perdido su papel desde hace tiempo en el transporte humano entre el valle y la ciudad de Mexicali.

24 Los ejidos de la zona compartida pueden ser evaluados a través de un estudio usando la teoría de los ensambles difusos, porque al mismo tiempo pueden estar dentro de una u otra zona. 


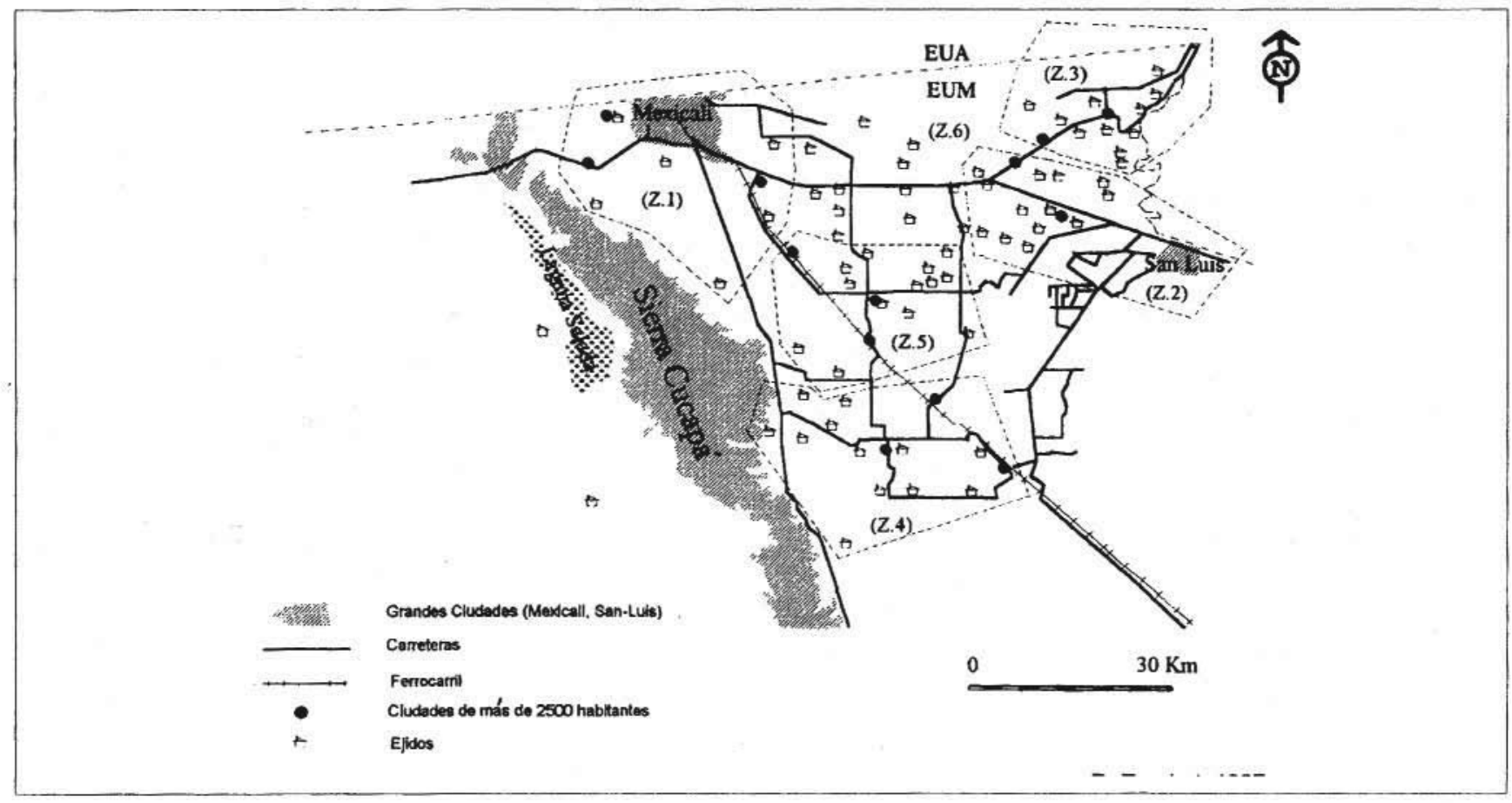

Figura 5. Zonificación ejidal. 
CUADRO 6. El sector primario y la cercanía a las grandes ciudades.

\begin{tabular}{lrccc}
\hline Localidades & Población & $\begin{array}{c}\text { Sector } \\
\text { primario }(\%)\end{array}$ & D1 (km) & D2 (km) \\
\hline A.Oviedo Mota & 6279 & 71.43 & 55 & - \\
Coahuila & 5349 & 65.54 & 75 & - \\
Hermosillo & 4974 & 65.29 & 65 & 20 \\
Paredones & 3510 & 65.22 & 58 & 27 \\
G. Victoria & 10816 & 60.76 & 60 & \\
Benito Juárez & 4242 & 56.66 & 55 & 32 \\
Cd. Morelos & 6031 & 56.65 & 65 & 23 \\
Delta & 5040 & 55.58 & 42 & - \\
Nuevo León & 3073 & 54.83 & 38 & - \\
Progreso & 2547 & 54.78 & 12 & - \\
Santa Isabel & 5624 & 47.74 & 10 & - \\
Puebla & 5240 & 47.22 & 15 & - \\
Michoacán de Ocampo & 2659 & 28.62 & 22 & 65 \\
San Felipe & 9263 & 27.22 & $*$ & $*$ \\
\hline
\end{tabular}

FUENTE: Las dos primeras columnas provienen del Censo de población y vivienda, 1990 (INEG1, 1990). Las dos últimas son una estimación de la Base Espacial Basemex.1. CREDAL-IS-UABC, 1996.

* Fuera de nuestro estudio.

D1 Estimación de distancia hacia Mexicali.

D2 Estimación de la distancia hacia Sán Luis Rio Colorado.

frecuencia de los desplazamientos. Con una tasa de motorización que está en el mismo nivel que la de Irlanda - aunque los salarios estén fuera de toda comparación-, el automóvil facilita la duración de esta opción residencial (Depuis, 1996).

La organización residencial rural perturbada en sus fundamentos después de la crisis que sacudió la actividad agrícola, ve sus potencialidades renovadas con el arranque del ciclo maquilador. La nueva reorganización en función de los nuevos datos de la economía regional, hace que hoy ese espacio tierra adentro ya no sea inerte, sino "un anexo" a la gran ciudad urbana. $\mathrm{La}$ interpenetración territorial y la confusión de las funciones parecen ser las características del nuevo modelo de integración rural. La vocación espacial, lejos de asegurar su reproductibilidad en la unidad funcional, se diversifica y se moldea en todas las formas del desarrollo 
regional. La supremacía del modelo residencial urbano, hace que el desplazamiento laboral sea visto como una suerte para asegurar ingresos. Sobre ese espacio fronterizo, los salarios, el bienestar familiar y la integración social evolucionan en un espectro espacial a intervalos reducidos. La vecindad de diversos modelos de desarrollo resalta el contraste de la contradicción espacio-social.

\section{DE LA VOCACIÓN ESPACIAL A LA INTEGRACIÓN EN LA DIFERENCIA: DE LA GLOBALIDAD A LA ORIGINALIDAD}

La integración de las localidades del valle de Mexicali en el sistema residencial regional es un hecho establecido en primer lugar por la socialización de los medios de producción agrícola. La confirmación y la legitimación de ese modelo de producción, sin embargo, se hizo de manera paralela al crecimiento de la demanda internacional del algodón. La caída del precio de la fibra natural marcó el fin del modelo residencial mantenido por la vocación agrícola. La conservación de la vivienda rural durante y después de la crisis es problemática, es lo menos que podemos decir. Problemática, en el sentido de que el esquema funcional ya no es el motor del desarrollo económico y social, menos aún su reconversión agrícola actual. El peso regional de las recombinaciones económicas y laborales tienen una incidencia más fuerte que la regeneración del espacio rural únicamente por su potencial tradicional. Las características de la nueva producción agrícola mayoritaria son de dos órdenes: la maximización de los subsidios y el subcontrato del trabajo precario por las poblaciones migrantes (Zabin, 1991:337-355).

La segmentación del mercado del trabajo rural en Mexicali (Moreno Mena, 1994:31-60) en función de la fluctuación de la demanda laboral, pero sobre todo a consecuencia de la adopción de la regresión salarial, ${ }^{25}$ acabó sólo por trazar el límite corporativista y la jerarquización de un profesionalismo agrícola de precariedad. El "corredor" del trabajo agrícola temporal y precario que nació en los confines de los estados expulsores de población, está ahora lejos de acabar su carrera sobre el valle de Mexicali. como era el caso durante el periodo de los años cuarenta y cincuenta.

$\mathrm{El}$ análisis de la estructura intema de las redes residenciales del valle de Mexicali acredita la idea del centralismo como base de la mayoría de las teorías rurales. Esa idea de centralismo es fundamental, porque sirve de base a buen número de enfoques en el ramo de la conceptualización de

${ }^{25}$ Como consecuencia de la perennización del trato social en una óptica altamente compttitiva (producción de hortalizas) o continuamente restrictiva (subsidios de granos y de fibra). 
la vocación espacial y el ordenamiento rural. La quiebra del sistema residencial atraído por la actividad agrícola, ¿podría ser la señal de la quiebra del modelo de integración rural?

Es verdad que la atracción de mano de obra local por la actividad agrícola ha disminuido a lo largo de los años, en beneficio de la migración y el desplazamiento laboral cotidiano. El volumen del éxodo neto hacia las grandes ciudades, si tomamos en cuenta la variación de la población rural, no presenta una tendencia francamente masiva. La regulación del éxodo rural hacia la ciudad es un hecho real. Pensamos que esa regulación del flujo humano se hace a partir del mejoramiento de las condiciones y de los medios individuales para el desplazamiento y el alza de la especulación inmobiliaria sobre los terrenos y el patrimonio urbano construido. El abandono relativo del trabajo agrícola por la población nativa local, contribuyó ciertamente a reforzar la segmentación del mercado laboral agricola a través del subcontrato de trabajadores no nativos en las ramas más precarias. Si esa afirmación es verdad en su totalidad, por lo menos hay que matizarla en función del componente y de la diversidad de la población migrante.

La diversidad de la población migrante, en función de sus características demograficas, su estrategia de gestión de la estancia laboral y los intereses dejados atrás, le da un comportamiento complejo en el mercado de trabajo. La participación de la fuerza laboral inmigrante en el sector primario es de $9.16 \% ;{ }^{26}$ para el grupo de migrantes que están en el mismo lugar desde bace un tiempo ( 0 a 9 años), esa misma tasa baja a $1.87 \%$, (apuntemos que la participación de la población nativa en el mismo sector es de $8.25 \%$ ). Esas cifras traducen la débil representación de la población recién instalada o la que llega a los lugares de la actividad agrícola, sin embargo, está mejor representada en los otros sectores. ${ }^{27}$ Esa débil representación puede encontrar su explicación en el cierre con el tiempo del sector de la tierra a los recién llegados, ${ }^{28}$ porque esa tasa de participación aumenta en relación con la duración de sedentarización (Sánchez, 1994:90-94).

Las hortalizas que requieren personal conocedor del modo de cultivo, hacen que los productores prefieran una mano de obra local más capacitada para la realización de esa tarea (Moreno Mena, 1994:48). El nuevo empuje en

\footnotetext{
26 Según una estimación a partir de 1865 , muestras de la Encuesta demográfica de Baja california de 1990 ( cit. en Sánchez, 1994).

$27.84 \%$ en el secundario y $7.66 \%$ en el terciario.

28 Esta encuesta probablemente no representó bastante la franja de la población agrícola de verano, en particular en las labores de algodón, que según varios autores citados, dan estimaciones mucho más importantes.
} 
el cultivo del algodón concentra actualmente la mayoría de los trabajadores en condiciones laborales y de salubridad lamentables. Debido a su precaria situación laboral, la agricultura del valle de Mexicali es incapaz de reflejar condiciones de vida decentes para los obreros agrícolas, y aun para una mayoria de productores.

Si examinamos a la luz del Censo de población y vivienda de 1990 las condiciones de la vivienda rural, observamos un dominio de la composición de las viviendas de dos cuartos. El volumen de las casas que cuentan con un solo cuarto es también muy importante. La composición por número de cuartos no parece tener correlación con el trayecto que separa los ejidos de los centros urbanos correspondientes (ver figura 6). Apuntemos, sin embargo, una frecuencia más o menos elevada de casas con un solo cuarto situadas alrededor de la ciudad de Mexicali. Esa afirmación es en parte válida para la ciudad de San Luis Río Colorado. En la totalidad, incluyendo las "ciudades pequeñas" en el área rural, observamos una mayor concentración de los ocupantes por casa en los perímetros rurales que en los perímetros urbanos de la ciudad de Mexicali (ver figura 7),

Ese fenómeno de concentración se puede traducir también en la tasa de cuartos por casa que favorece más al área urbana (ver figuras 7 y 8). Los materiales de construcción empleados en las residencias rurales y urbanas presentan tasas similares, a excepción de la cubierta por suelo, que es más elaborada en la zona urbana que en la rural (Toudert, 1997:19-21). La situación de la vivienda rural localizada en el área de débil tensión urbanística y menor presión especulativa, es paradójicamente menos ventajosa. Esa diferencia espacial puede encontrar una explicación en la falta de inversión familiar destinada a la vivienda, enfatizada por el desequilibrio salarial entre el ingreso entre la zona urbana y la zona rural. ¿No sería la masificación de la casa rural una respuesta familiar a la crisis? La falta de datos no permite ahondar en esa pregunta, pero podemos suponer, en función de las observaciones hechas en otros lugares, que la crisis del trabajo rural y la del trabajo en general redujo la salida de los hijos de la casa familiar a temprana edad para formar su propio hogar (Mendras, 1988:256-260).

La cuestión de la integración rural nos parece, pues, como un problema complejo y minado. Complejo en el sentido de que los actores y los espacios son variados y tienen diferentes niveles de inserción. Minado en cuanto que los intereses puestos en juego son mayores y las fugas numerosas. Más allá de la generosidad del discurso sobre la integración espacial se hace la pregunta: de mantener o no los equilibrios que han prevalecido ya desde hace mucho tiempo, ia quién vamos a cobrar el recibo de ese gesto de integración territorial? 
El deber de la solidaridad que está en el origen de todas nuestras repúblicas modernas, fue maltratado por la lógica de los extremos. El neoliberalismo, y sobre todo, su tendencia - la más "sanguinaria"- a ocultar al Estado hasta las más profundas funciones de regalías, paralelamente demostró su incapacidad en producir los cambios democráticos necesarios en una sociedad civil pauperizada.

En dondequiera se cuestiona sobre la rehabilitación del ciudadano y su responsabilidad de hacerse cargo de sí mismo, sabiendo que la oposición de los espacios en todas sus dimensiones no beneficia al mundo rural, y menos al mundo urbano. La interconexión espacial es ahora una realidad, $y$ el interés que tienen los ciudadanos sobre el medio ambiente ha sido fundamental para la difusión de las ideas de continuidad y de un desarrollo duradero. La disposición de los recursos, una mejor cobertura espacial y residencial en eI respeto de las interacciones de convivencia y la capacidad de carga del medio ambiente, son las nuevas fuerzas de un despliegue espacial. La toma de conciencia de su medio, de sus capacidades de superación, son dos elementos claves en la búsqueda y la construcción de una vocación espacial justa y equitativa (Sachs, 1993). Salir del engranaje de la disposición rural hacia un concepto más abierto del desarrollo integral, es una prueba dada a la sociedad rural para estimular su fuerza de empresa reducida a nada después de los últimos años de depresión; es también una invitación a la población urbana sobre ideas diferentes de la alteridad de las políticas duales. Interconexión territorial, interconexión y transferencias de ideas y de acciones, redistribución de las oportunidades en el respeto de los valores comunes, son los nuevos factores que pueden conciliar al hombre y su espacio. La reconquista del espacio perdido en toda su dimensión humana, cultural y ambiental del valle de Mexicali, permanece aún como una tarea compleja. La promiscuidad de los modelos distintos del desarrollo y la diferencia de las condiciones laborales y del bienestar social (exagerados por la situación fronteriza) continuarán imprimiendo todavía a los territorios las fracturas espaciales ya demasiado visibles.

El debate sobre los ajustes espaciales en términos de revaluación espacial hacia lo alto o hacia lo bajo para realizar los equilibrios que suponen legitimizar un proceso largo de desarrollo equitativo, ha demostrado sus límites de integración. Aparte de su lado consensual, ha evaluado sobre todo los espacios hacia abajo, sirviendo los propósitos de las fuerzas del mercado, que pone como condición la revisión de las políticas sociales como preámbulo de la perennización y el reparto del ciclo de desarrollo. Para los representantes de la globalización, los territorios ( $\mathrm{y}$ las naciones) más avanzados deben contribuir al desarrollo de los 


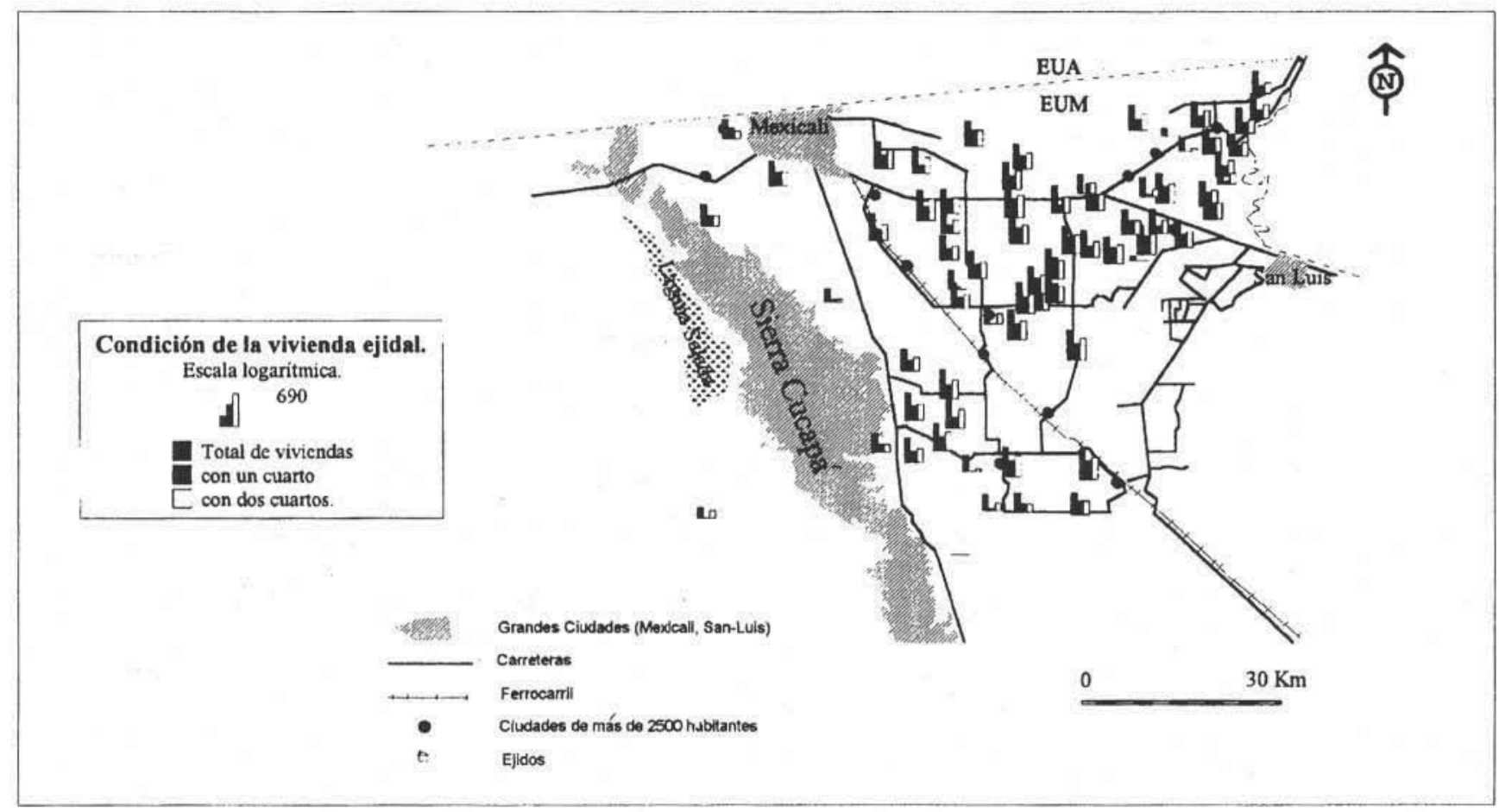

Figura 6. Composición de las viviendas ejidales por número de cuartos. 


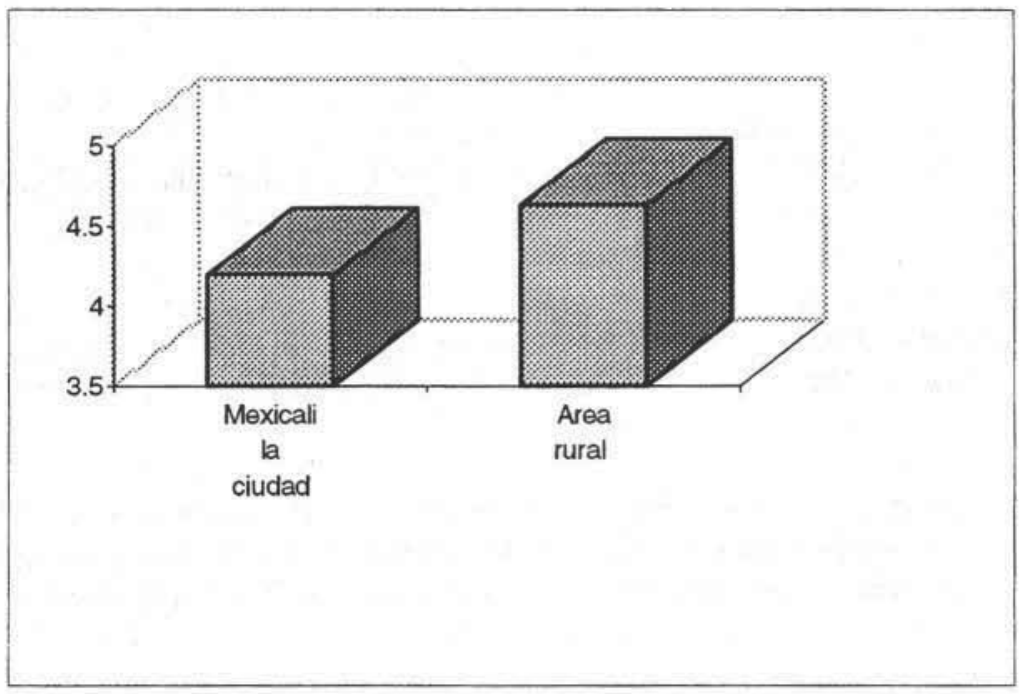

Figura 7. Promedio de ocupantes por vivienda.

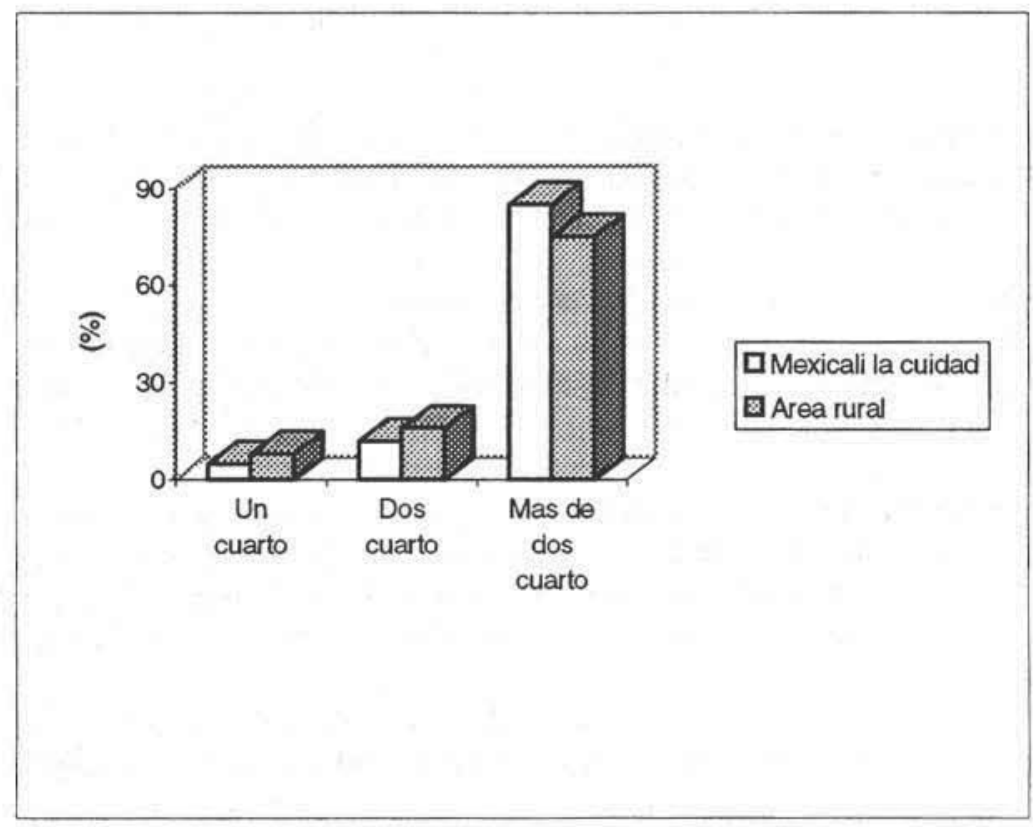

Figura 8. Composición de las viviendas por número de cuartos. 
espacios marginales por el abandono de política social. Ésta y otras tesis crean la popularidad de esta corriente dentro de los sectores de los países en vías de desarrollo más abiertos al progreso.

En Mexicali, como en cualquier otra parte, se cuestiona sobre la descentralización y la democracia local, así como acerca de la participación de los ciudadanos en la elaboración de las políticas de desarrollo por medio de todos los artificios de atracción y de perennización de las inversiones capaces de provocar el despegue de los espacios marginales sobre ciclos de desarrollo duradero. ¿Debemos de conceder a la localidad la posibilidad de definir su política de desarrollo?

Tener los medios de una política de desarrollo vuelve a poner en manos de las localidades la posiblidad de escoger su política fiscal de acuerdo con sus especificidades y sus necesidades. La regulación de estas políticas fiscales en medio de un dispositivo de control de la competencia interlocal en atención a una carta de principios sobre el respeto de los fundamentos de la interconexión regional y de sus otras ramificaciones nacional e internacional, ecoloca de nuevo al Estado en su papel de regulador de los flujos de intercambio como actor de desarrollo local que no se puede eludir. La intervención del Estado en los espacios marginales ya no puede limitarse únicamente al reparto del producto nacional de solidaridad, sino que debe emprender políticas de desarrollo importantes y audaces. La intervención voluntarista del Estado, de común acuerdo con los actores locales para la puesta en marcha de otra política de desarrollo puede hacerse, pot ejemplo, alrededor de la declaración de esta área rural como zona de desarrollo prioritario, donde va a venir a dar un tren de medidas, que pueden partir del aligeramiento fiscal hasta las ayudas subsidiarias de instalación: es una nueva zona franca en el interior de la zona franca matriz. La colocación de esa zona franca intema y temporal para que tenga una oportunidad de éxito, debe evitar, sobre todo, los errores del pasado; es decir, ver la salvación del mundo rural únicamente en la agricultura; dar una nueva oportunidad a esas localidades rurales del valle de Mexicali en función de la disponibilidad de recursos, la originalidad de las recombinaciones y las múltiples posibilidades de inserción en las redes locales y regionales. Todo este dispositivo debe basarse desde el principio en un conjunto de inversión. La modernización de la red de carreteras, del ferrocarril y de las telecomunicaciones, es una condición necesaria para acompañar ese desarrollo. Una vez más, una zona franca prioritaria y temporal no constituye de ninguna manera una nueva frontera, menos aún el nacimiento de un nuevo desfasamiento espacial. Dicho esto, los resbalones y los riesgos de otra polarización se deben tomar en cuenta seriamente. 


\section{EN CONCLUSIÓN.}

Nos parece necesario atraer la atención sobre el hecho de que la era del desarrollo rural, usando la producción agrícola aquí en el valle de Mexicali, es ahora una historia acabada. Querer prenderse de ese ideal equivale a perder la oportunidad de integración del modelo residencial rural que realmente la conserva aún.

A partir de este trabajo, se resalta que el asunto de integración nural es complejo, mas no imposible. Es además indispensable, porque ya no podemos ignorar espacios que son mucho más grandes que nuestro espacio vivo reducido como una piel de zapa en nuestras ciudades urbanas. $\mathrm{La}$ integración rural boy más que nunca, ya no puede ser el monopolio de un grupo de actores, es un movimiento de sociedad para la rehabilitación de un patrimonio común. Lejos de un gesto de nostalgia pasajero, es el derecho de los ciudadanos de vivir plenamente su espacio.

\section{BIBLIOGRAFÍA}

Agenda Estadística del Municipio de Mexicali, Baja California. 1990. Cuadernos de estudios y proyectos, núm. 3 .

ANGUIANO, Maria Eugenia. 1995. Agricultura y migración en el valle de Mexicali, Colef, Tijuana, B.C.

COLLIN DELAVEAUD, Anne y Djamel Toudert. 1995. "L'apport d'une simple image satellite dans l'étude d'une ville: Le cas de Mexicali", Journnées de la Télédétection de l'AUPELF-AUREF, Université de l'Ausanne, Bélgica.

DEPUIS, Gabriel. 1996. "La evolución de la ciudad y las ciudades del futuro", Seminario IIS-UABC, diciembre.

INEGI. 1991. Censo de población y vivienda ejidal.

- 1992. "Baja Califomia: El perfil sociodemográfico", XI censo general de población y vivienda, 1990.

- 1994. Anuario estadístico del estado de Baja California.

LARCENA, Daniel, et. al. 1996. "Les Paysages de la Crau par Télédétection: Approche Méthodologique pour l'étude des Paysages", EHESS, Marseille.

MENDRAS, H. 1988. Sociologie des Ruraux, Enceclopaedia Universalis, vol. 16.

MORENO Mena, José A. 1994a. "La conformación histórica del mercado de trabajo agrícola en el valle de Mexicali", Estudios fronterizos, núm.33, enero-junio, IIS-UABC, Mexicali, B.C. 
- 1994b. "Una aproximación a las características actuales de la mano de obra agricola en el valle de Mexicali", Estudios fronterizos, núm. 34 , julio-diciembre, IIS-UABC.

PORTAIS, Michel, 1995. "Frontiére nord-sud et Modèles Spatiaux: Valle de Mexicali-Imperial Valley", La frontiére Mexique-Etats-Unis (dir. J.R. Mouroz y P. Gondard), IHEAL, París.

RANFLA González, Arturo. 1991, "Mexicali contemporáneo, 19501990", en Mexicali: Una historia. Instituto de Investigaciones Históricas, UABC.

SACHS, I. 1993. L'écodéveloppement Stratégies de Transitions vers le XXe siécle. Internatives Économiques, ed. Syros, Paris.

SÁNCHEZ, María Dolores. 1994. "Dinámica migratoria, educación y mercado de trabajo; el caso de Baja California", tesis de maestria en Desarrollo urbano y regional, IIS-UABC.

SCHMIDT, Samuel. 1994. "Migración mexicana a Estados Unidos. Notas para una solución", Estudios fronterizos, núm. 33, enero-junio, IIS-UABC.

STAMATIS Maldonado, Martha. 1993. "Los contratos de producción en el noroeste de México: El valle de Mexicali a fines de la década de los ochenta", Estudios fronterizos, núm. 30, enero-abril, IIS-UABC, Mexicali, B.C.

TOUDERT, Djamel. 1995a. Les Changements Agricoles dans el Valle de Mexicali, Men de DEA, IHEAL-CREDAL, París.

- 1995b. 'Les Mutations de l'agricultures Mexcaine: Les Cas des Productions Pivots á el Valle de Mexicali", mesa redonda sobre la frontera, México-EUA, CREDAL-ORSTOM, París.

- 1997. "El agua, el campo y la ciudad, la emergencia de un nuevo conflicto regional", ponencia presentada en el Seminario de Investigaciones sobre la Gestión Eficiente del Agua en Sistemas de Riego Regionales, Colef-Mexicali, Mexicali, B.C.

ZABIN, Carol. 1997. "US-Mexico Economic Integration Labor Relations and Organisation of Work in California and Baja Califomia Agriculture", Economic Geography, vol. 73, núm. 3, julio. 